\title{
Implications of recovery procedures on structural and rheological properties of schizophyllan produced from date syrup
}

Hajar Jamshidian ${ }^{\text {a }}$, Seyed Abbas Shojaosadati ${ }^{a}, *$, Seyed Mohammad Mousavi a , Mohammad Reza Soudi b, Francisco Vilaplana ${ }^{\mathrm{c}, *}$

${ }^{a}$ Biotechnology Group, Chemical Engineering Department, Tarbiat Modares University, 14115-143 Tehran, Iran

${ }^{\mathrm{b}}$ Microbiology Department, Faculty of Biological Sciences, Alzahra University, 1993891176 Tehran, Iran.

${ }^{c}$ Division of Glycoscience, School of Biotechnology, KTH Royal Institute of Technology, AlbaNova. University Centre, SE-10691 Stockholm, Sweden.

\begin{abstract}
This study investigates the effects of different recovery procedures on high molar mass schizophyl-lan produced by Schizophyllum commune using low value agricultural residues. Recovered extracellular polysaccharides (EPSs) were compared in terms of purity, sugar composition, degree of branching, molec-ular weight, and rheological properties. Performing different recovery methods, such as re-dissolving in water and re-precipitation with ethanol on produced EPS, provided schizophyllan with purity similar to the commercial grade. Besides, Freeze-thawing cycles allowed the fractionation of schizophyllan based on branching degree and solubility. The EPSs with higher purity and lower degree of branching (less conformational flexibility) showed higher viscosity. This study evidences the possibility of producing EPSs with excellent rheological properties using low value agricultural side products. Furthermore, our results demonstrate the importance of recovery methods for tailoring the purity, molecular structure and macroscopic properties of the produced polysaccharides for specific applications.
\end{abstract}

Keywords: Schizophyllan, Exopolysaccharide (EPS), Recovery procedure, Rheological properties, Molecular structures. 